\title{
Associations of childhood adiposity and changes in adiposity status from childhood to adulthood with pregnancy hypertension
}

Ye HE, MM, Menzies Institute for Medical Research, University of Tasmania, Hobart, Australia

Jing TIAN, PhD, Menzies Institute for Medical Research, University of Tasmania, Hobart, Australia

Leigh BLIZZARD, PhD, Menzies Institute for Medical Research, University of Tasmania, Hobart, Australia

Wendy H. ODDY, PhD, Menzies Institute for Medical Research, University of Tasmania, Hobart, Australia

Terence DWYER, MD, Menzies Institute for Medical Research, University of Tasmania, Hobart, Australia; The George Institute for Global Health, University of Oxford, Oxford, UK.

Alison J. VENN, PhD, Menzies Institute for Medical Research, University of Tasmania, Hobart, Australia.

Conflicts of Interest: The authors report no conflicts of interest

Funding: This study was supported by grants from the National Health and Medical Research Council (grants 211316 and 544923).

Previous presentation: The abstract was presented at the XXIII Annual Congress of Perinatal Society of Australian and New Zealand from 17th-20th March 2019 in Gold Coast, Australia. 


\section{Corresponding Author:}

Professor Alison J. Venn

Menzies Institute for Medical Research, University of Tasmania

17 Liverpool Street, Hobart, Tasmania 7000, Australia

Telephone: 61-3-6226-7706

Fax: 61-3-6226-7704

E-mail: Alison.Venn@utas.edu.au 
Condensation: Childhood abdominal obesity was associated with pregnancy hypertension independent of childhood and adult body mass index.

Short Title: Childhood adiposity and pregnancy hypertension

\section{A. Why was the study conducted?}

To investigate the associations between different adiposity measures in childhood, and change in adiposity status from childhood to adulthood, with pregnancy hypertension

\section{B. What are the key findings?}

Childhood abdominal obesity was associated with pregnancy hypertension independent of childhood and adult body mass index. Women who were persistently overweight/obese or abdominally obese since childhood had the highest risk of pregnancy hypertension.

\section{What does this study add to what is already known?}

Our article shows the importance of childhood abdominal obesity, in addition to BMI, as an indicator of future cardiometabolic risk including pregnancy hypertension, and the importance of preventing childhood obesity.

Key words: abdominal obesity, body mass index, childhood, pregnancy hypertension, waistto-height ratio 


\begin{abstract}
Background: Pregnancy hypertension is a significant cause of maternal morbidity and mortality. Elevated childhood body mass index (BMI) has been identified as a risk factor for pregnancy hypertension, however, no study to date has reported the role of childhood abdominal obesity and change in adiposity status from childhood to adulthood with pregnancy hypertension.
\end{abstract}

Objective(s): To investigate the associations between different adiposity measures in childhood, and change in adiposity status from childhood to adulthood, with pregnancy hypertension.

Study Design: The study followed-up 985 girls who participated in the 1985 Australian Schools Health and Fitness Survey when aged 9-15 years and were ever pregnant at followup in 2004-2006 and/or 2009-2011 when they were aged 26-41 years. Childhood anthropometric measures included height, weight, waist and hip circumferences. At followup, pregnancy hypertension was self-reported by questionnaire and defined as having high blood pressure during pregnancy or due to pregnancy. Overweight and obesity in childhood were defined according to age and sex-specific international standard definitions for BMI. Childhood abdominal obesity measures included waist circumference, waist-to-hip ratio and waist-to-height ratio (WHtR) with abdominal obesity defined as WHtR $\geq 0.5$. Generalized estimating equations for log binomial regression were used to estimate relative risks (RRs) and 95\% confidence intervals (CIs) adjusting for age, area-level disadvantage in childhood, parity and adult occupation.

Results: Ever having pregnancy hypertension at follow-up was reported by $141(14.3 \%)$ women. Increased childhood BMI was associated with a higher risk of later pregnancy hypertension, but this risk did not persist after adjustment for adult BMI. Childhood 
abdominal obesity was associated with a significantly increased risk of pregnancy hypertension independent of both childhood and adult BMI. Girls with one standard deviation higher waist circumference $(7.51 \mathrm{~cm})$, waist-to-hip ratio $(0.06)$ and WHtR $(0.04)$ had a $29 \%$ (95\% CI:1-65\%), 20\% (95\% CI:3-41\%) and 24\% (95\% CI: 6-41\%) greater likelihood of pregnancy hypertension, respectively. Compared with girls who were not abdominally obese, those who were had more than twice the risk of having pregnancy hypertension $(R R=2.21$, 95\% CI:1.09-4.48).

The associations of adiposity with pregnancy hypertension were stronger in participants with persistently high adiposity status from childhood to adulthood than those with high adiposity status only in adulthood. Compared with participants with persistently normal BMI or normal WHtR, those who were overweight/obese or abdominally obese only in adulthood had an increased risk of pregnancy hypertension $(\mathrm{RR}=1.49,95 \% \mathrm{CI}: 1.10-2.02$ and $\mathrm{RR}=1.43,95 \%$ CI:0.92-2.25). Participants who were persistently overweight/obese or abdominally obese from childhood to adulthood had a significantly higher risk of pregnancy hypertension $(\mathrm{RR}=2.06,95 \% \mathrm{CI}: 1.29-3.29$ and $\mathrm{RR}=3.09,95 \% \mathrm{CI}: 1.54-6.20)$. All $p$-values $<0.05$.

Conclusion(s): Childhood abdominal obesity was associated with later pregnancy hypertension independent of childhood and adult BMI. Women who were persistently overweight/obese or abdominally obese since childhood had the highest risk of pregnancy hypertension.

Key words: abdominal obesity, body mass index, childhood, pregnancy hypertension, waistto-height ratio 


\section{Introduction}

Hypertension affects an estimated $10 \%$ of all pregnancies and is the most frequently identified medical problem during pregnancy. Hypertensive disorders of pregnancy include both pregnancy-induced (gestational hypertension and preeclampsia) and pre-existing (chronic hypertension) conditions and are associated with a higher risk of cardiovascular events later in life. ${ }^{1}$

Being overweight or obese puts a woman at risk of developing pregnancy hypertension. The risk of preeclampsia doubles with each 5-7 unit increase in pre-pregnancy body mass index $(\mathrm{BMI}){ }^{2}$ Abdominal obesity has also been associated with pregnancy hypertension. The results from one Australian cohort indicated that every $1 \mathrm{~cm}$ increase in waist circumference was associated with a $4 \%$ increased risk of pregnancy hypertension. ${ }^{3}$ Another case-control study indicated that waist circumference was a better predictor of pregnancy hypertension than BMI. ${ }^{4}$ Despite the known detrimental effect of adult adiposity on pregnancy hypertension, the relationship with childhood obesity, especially childhood abdominal obesity with pregnancy hypertension is less well understood.

To our knowledge, only two studies have reported a longitudinal relationship between childhood obesity and pregnancy hypertension, and childhood BMI was the only predictor. ${ }^{5,6}$ The 1958 British birth cohort study included 4,681 girls and reported that being overweight or obese at the age of 7 years increased the risk of self-reported hypertension in pregnancy before age 33, but this risk did not persist after adjustment for adult BMI. ${ }^{5}$ More recently, a report based on 703 participants in the USA Bogalusa Heart Study showed that elevated childhood BMI was a significant risk factor for self-reported pregnancy hypertension without considering adult BMI. 
Therefore, the aim of our present study was to examine the association between different adiposity measures in childhood and changes in adiposity status from childhood to adulthood, with pregnancy hypertension.

\section{Materials and Methods}

\section{Participants}

The Childhood Determinants of Adult Health (CDAH) Study is a follow-up of 8,498 children including 4,191 girls aged 7-15 years who participated in the 1985 Australian Schools Health and Fitness Survey (ASHFS), a nationwide sample of Australian school children. ${ }^{7}$ In 1985, all children had physical assessments and those aged 9, 12 and 15 years had blood pressure measured. During 2002-2004, a total of 3,412 female participants were traced and 2,734 enrolled to participate in the CDAH Study (enrolment) (Figure 1). During 2004-2006, the first follow-up (CDAH-1) of those enrolled was conducted when participants were aged 2636 years. Of the 1,017 women who reported having ever been pregnant and answered questions about pregnancy hypertension in the questionnaire, 735 attended one of 34 study clinics held around Australia for physical measurements. The second follow-up (CDAH-2) was conducted during 2009-2011 when participants were aged 31-41 years and 901 reported having ever been pregnant women and completed the same questions about pregnancy hypertension. A total of 1,324 women who were ever pregnant and answered the pregnancy hypertension questions at CDAH-1 and/or CDAH-2 were included in the analysis reported here (423 women only participated in CDAH-1, 307 women only participated in CDAH-2 and 594 women participated in both CDAH-1 and CDAH-2).

The study was approved by the Southern Tasmania Health and Medical Human Research Ethics Committee. Written informed consent was obtained at childhood from parents and obtained at each follow-up from participants. 


\section{Childhood adiposity measures}

BMI, calculated as weight $(\mathrm{kg}) /$ height $(\mathrm{m})^{2}$, was derived from measured height and weight with weight status defined using international age- and sex-specific cut-points. ${ }^{8}$ Waist and hip circumference was measured to the nearest $0.1 \mathrm{~cm}$. Waist-to-hip ratio was calculated by dividing waist by hip circumference. Waist-to-height ratio (WHtR) was calculated by dividing waist circumference by height. Abdominal obesity was defined as WHtR $\geq 0.5{ }^{9}$ Childhood BMI and waist circumference $\mathrm{z}$ scores were calculated based on age- and sexspecific standardization of the full childhood cohort.

\section{Adult adiposity measures}

At CDAH-1, weight, height, waist circumference and hip circumference were measured at study clinics for most participants. Some participants $(n=1,119)$ also self-reported their weight and height before measurements were taken to assess the accuracy of self-reported values. Participants who did not visit clinics self-reported their weight and height, and a correction factor was applied to adjust for error. ${ }^{10} \mathrm{BMI}\left(\mathrm{kg} / \mathrm{m}^{2}\right)$ was calculated from height and weight. WHtR was calculated from measured waist circumference and height at clinics.

Weight was self-reported at CDAH-2. Adjusted weight values were calculated using the correction factor applied at CDAH-1. Height was self-reported and adjusted as described above.

Adult BMI was categorized as normal $\left(\mathrm{BMI}<25 \mathrm{~kg} / \mathrm{m}^{2}\right)$, overweight $\left(25.0 \leq \mathrm{BMI} \leq 29.9 \mathrm{~kg} / \mathrm{m}^{2}\right)$ or obese $\left(\mathrm{BMI} \geq 30 \mathrm{~kg} / \mathrm{m}^{2}\right) .{ }^{11}$ Adult abdominal obesity was defined as $\mathrm{WHtR} \geq 0.5 .{ }^{12}$

\section{Pregnancy hypertension}


In CDAH-1 and CDAH-2, women were asked to answer 'yes' or 'no' to the question 'Have you ever been told that you have high blood pressure during pregnancy or due to pregnancy?' Pregnancy hypertension was recorded if they responded 'yes'.

\section{Covariate measures}

Information on sociodemographic characteristics was self-reported in childhood and followup including childhood age, parental education, parental smoking and childhood smoking experimentation, both childhood and adult area-level disadvantage ${ }^{13}$, alcohol consumption, physical activity and adult education level, smoking status, occupation and parity.

\section{Statistical analyses}

Taking into account the repeated measures of variables over CDAH-1 and CDAH-2, logbinomial models with generalized estimating equations (GEE) were used to estimate relative risk (RR) for associations between childhood adiposity measures and change in adiposity status from childhood to adulthood with pregnancy hypertension. Subsample analysis which considered the effect of adult abdominal obesity on pregnancy hypertension was conducted among those who participated in ASHFS and CDAH-1 clinics.

Covariates remaining in the final model were variables which associated with the exposure and the outcome and resulted in more than $10 \%$ change in the coefficient of the study factor.

The following sensitivity analyses were conducted. First, we excluded those who reported 'yes' to ever having pregnancy hypertension in CDAH-1 but 'no' in CDAH-2. Second, since multiple births and high childhood blood pressure may be associated with increased risk of pregnancy hypertension, ${ }^{14,15}$ we restricted our sample to women who had singleton pregnancies and those who had measured childhood systolic (SBP) and diastolic blood 
pressure (DBP). Third, inverse probability weighting was used to account for missing data at follow-up, with multiple imputation of incomplete baseline data. ${ }^{16}$

Finally, to examine if there is a difference in risk of pregnancy hypertension associated with adiposity during different time periods of growth, we repeated the analyses by stratifying childhood age before or after 12 years.

All analyses were performed using STATA software, version 15.0 (Stata Corp., College Station, TX); A p-value $<0.05$ was considered statistically significant.

\section{Results}

\section{Participant characteristics}

This study included 1,324 women who had ever been pregnant and completed hypertension questions at follow-ups; 594 of them completed both follow-ups; 423 participated in CDAH1 only; and 307 women participated in CDAH-2 only. Participants who had missing data on confounders $(n=339)$ were excluded, leaving 985 women for the final analysis.

Characteristics of participants in childhood and adulthood are shown in Table 1. In childhood, 10 (1.0\%) girls were obese, 77 (7.8\%) were overweight as defined by BMI cutoffs, and 47 (4.8\%) had abdominal obesity as defined by WHtR category. The mean age at CDAH-1 was 32.8 years and 37.5 years at CDAH-2. There were $111(13.8 \%)$ and $68(10.6 \%)$ women who reported experiencing pregnancy hypertension in CDAH-1 and CDAH-2. Among them 21 women reported having had pregnancy hypertension in CDAH-1 but not in CDAH-2.

Compared with those who did not participate in CDAH-1 or CDAH-2, those who did participate in CDAH-1 and/or CDAH-2 were older in childhood (12.1 vs 10.5 years; 
$P=0.000)$, had greater BMI (18.8 vs $\left.18.2 \mathrm{~kg} / \mathrm{m}^{2} ; P=0.000\right)$ and waist circumference (64.1 vs $62.1 \mathrm{~cm} ; P=0.000)$, lower WHtR ( 0.42 vs $0.44 ; P=0.000)$ and waist-to-hip ratio ( 0.80 vs 0.82 ; $P=0.000)$, and were less likely to be obese $(1.0 \%$ vs $1.8 \% ; P=0.001)$ or abdominally obese $(4.8 \%$ vs $7.9 \% ; P=0.001)$.

\section{Pregnancy hypertension}

As shown in Table 2, after adjustment for age, area-level disadvantage in childhood, parity and occupation in adulthood, childhood BMI and abdominal obesity measures were all associated with an increased risk of pregnancy hypertension (Model 1). To determine whether the observed associations of childhood abdominal obesity measures with pregnancy hypertension were independent of childhood BMI, we further adjusted for childhood BMI (Model 2), the associations between childhood abdominal obesity measures and pregnancy hypertension remained significant. Model 3 included additional adjustment for adult BMI to investigate whether associations with childhood BMI and abdominal obesity measures were independent of adult BMI. Childhood BMI was no longer associated with pregnancy hypertension after adjustment for adult BMI (Model 3). However, associations remained for childhood abdominal obesity. Girls with one standard deviation higher waist circumference $(7.51 \mathrm{~cm})$, waist-to-hip ratio $(0.06)$ and $\mathrm{WHtR}(0.04)$ reported $29 \%(95 \%$ confidence interval [CI]:1-65\%), 20\% (95\% CI:3-41\%) and 24\% (95\% CI:6-43\%) greater likelihood of pregnancy hypertension. Compared with non-abdominally obese girls, those who were abdominally obese in childhood had more than twice the risk of having pregnancy hypertension $(\mathrm{RR}=2.21,95 \% \mathrm{CI}: 1.09-4.48)$. In the subsample of participants with measured waist circumference and height at the CDAH-1 clinics (Supplemental Table 1), the associations of childhood abdominal obesity with pregnancy hypertension persisted after adjusting for adult abdominal obesity ( $R R=2.15,95 \% \mathrm{CI}: 1.10-4.20)$. 


\section{Influence of adiposity change from childhood to adulthood}

The relative risk of pregnancy hypertension by BMI and WHtR category change from childhood to adulthood is displayed in Table 3. Compared with participants who had persistently normal BMI in childhood and adulthood, those who became overweight/obese reported a higher risk of pregnancy hypertension with a RR of 1.49 (95\% CI 1.10-2.02). The risk was highest for participants who were persistently overweight/obese from childhood into adulthood (RR=2.06; 95\% CI 1.29-3.29).

In the subsample of participants who had WHtR data in both childhood and adulthood $(\mathrm{N}=549), 19(3.5 \%)$ were abdominally obese in both childhood and adulthood and $31.6 \%$ of them had pregnancy hypertension (Figure 2). Compared with those who were not abdominally obese in childhood and adulthood $(68.3 \%$ of the subset, $11.5 \%$ with pregnancy hypertension), those who were persistently abdominally obese had a significantly higher risk of pregnancy hypertension ( $R R=3.09 ; 95 \% \mathrm{CI}$ : 1.54-6.20) (Table 3). Subjects who were not abdominally obese in childhood but who developed abdominal obesity in adulthood (27.5\%) also had a higher risk of pregnancy hypertension $(\mathrm{RR}=1.56 ; 95 \% \mathrm{CI} 1.00-2.43)$, but the significance of this association was attenuated following after adjustment for confounders.

\section{Sensitivity analysis}

Similar results were observed after excluding women who reported they had pregnancy hypertension in CDAH-1 but not in CDAH-2 $(n=21)$. When restricting the sample to women with singleton pregnancies, the risk of pregnancy hypertension $(\mathrm{RR}=2.55,95 \% \mathrm{CI} 1.28-5.09)$ remained significantly higher in those who were abdominally obese in childhood. A total of 415 girls aged 9, 12 and 15 years had blood pressure measured in childhood. The mean childhood SBP and DBP were similar in those with and without self-reported pregnancy hypertension in CDAH-1 (SBP: $110.4 \pm 14.8$ vs $109.0 \pm 11.8, P=0.50$; DBP: $65.4 \pm 12.2$ vs 
$66.8 \pm 10.8, P=0.43)$ and CDAH-2 (SBP: $112.6 \pm 12.7$ vs $108.5 \pm 11.8, P=0.06$; DBP: $67.8 \pm 11.1$ vs $66.1 \pm 10.6, P=0.38$ ). Further adjustment for childhood SBP and DBP in these participants did not substantially change the main results: the changes in the magnitude of significant estimates were within $17.2 \%$ (Supplemental Table 2). Sensitivity analyses to address loss to follow-up by using combined multiple imputation and inverse probability weighting produced similar patterns of results as the unweighted analyses: changes in the magnitude of significant associations ranging from -23.5-58.0\% (Supplemental Table 3).

When stratified by age, childhood BMI and BMI z score showed similar associations in 12 to 15 year-olds (RR: $1.07,95 \%$ CI 1.00-1.15; RR:1.21, 95\% CI 1.00-1.45) to those younger than 12 years (RR: $1.09,95 \%$ CI 0.99-1.19; RR: 1.20, 95\% CI 0.94-1.53). However, the observed statistically significant association in the 12 to 15 year age group did not persist after further adjustment for adult BMI. In a subsample analysis, a stronger association with pregnancy hypertension was found with abdominal obesity before age 12 than age 12-15 years after adjusting for adult abdominal obesity. RR was 1.59 (95\% CI 1.28-1.99) for per SD (0.04) increase in WHtR before age 12 and 1.05 (95\% CI 0.81-1.37) between 12 to 15 years.

\section{Comment}

\section{Main findings}

To the best of our knowledge, this is the first study to report the long-term associations of childhood abdominal obesity and change in body composition from childhood to adulthood with pregnancy hypertension. We found that childhood abdominal obesity was associated with an increased risk of pregnancy hypertension. This association was persistent across different abdominal obesity indicators and independent of childhood and adult BMI. Persistent overweight/obesity and abdominal obesity were associated with the highest risk of pregnancy hypertension. 


\section{Meaning of the findings}

Our finding that the association of childhood BMI with pregnancy hypertension was not independent of adult BMI was consistent with findings from the 1958 British birth cohort study ${ }^{5}$ and that overweight/obesity that is proximal to pregnancy is especially important for pregnancy hypertension risk. However, a higher risk was found for women who were persistently overweight/obese in childhood and adulthood than those who first became overweight/obese as adults. This result suggests that childhood adiposity is important because of the tendency for obesity to track from childhood into adulthood.

Although childhood abdominal obesity has been observed in several cross-sectional studies to be associated with cardio-metabolic risk in childhood ${ }^{12,17-19}$, the current understanding of the long-term effect of abdominal obesity on chronic conditions including pregnancy hypertension remains limited. Most studies from childhood have followed participants into adulthood and collected BMI due to ease of measurement. In our study, one SD of 0.04 unit increase in childhood WHtR was associated with 33\% greater likelihood of reporting pregnancy hypertension and was largely unchanged after adjusting for childhood and adulthood BMI. These results indicated a detrimental impact of childhood abdominal obesity on pregnancy hypertension, which was not modified by BMI. It has been suggested that early onset obesity may have higher risks of adverse outcomes in later life. ${ }^{20}$ Consistent with this we observed stronger associations with childhood abdominal obesity in girls younger than age 12 than in older girls. In the Bogalusa Heart Study, a stronger association of childhood BMI with pregnancy hypertension was reported between ages 12 to 17 than before age 12 . In our study, similar associations of childhood BMI with pregnancy hypertension were found in these younger than age 12 and age 12 to 15 after accounting for adult BMI. 
The exact mechanism by which childhood abdominal obesity influences risk of pregnancy hypertension remains unclear. Plausible mechanisms include the long-term adverse effects of excess childhood visceral fat on blood pressure, insulin resistance, inflammation upregulation, oxidative stress and endothelial dysfunction. ${ }^{21-24}$

\section{Strengths and limitations}

Some potential limitations of our study are acknowledged. The first is the measurement of pregnancy hypertension and that self-report may lead to misclassification. ${ }^{25,26}$ However, the prevalence of pregnancy hypertension in our study is similar to global prevalence. ${ }^{1}$ Beyond that, two validation studies to investigate the validity of self-reported pregnancy hypertension show good concordance of self-report with clinical records. ${ }^{25,27}$ Second is the loss to followup. We applied inverse probability weighting to account for missingness but these did not appreciably change the results. Third is the potential over adjustment of adult BMI and abdominal obesity measures. Our findings were for ever-pregnant women, but we were unable to account for their BMI and abdominal measures immediately prior to pregnancy. Women's weight or waist circumference may increase after pregnancy, however, over adjustment of adult anthropometric measures tend to favor our study results. Lastly, we cannot classify the category of pregnancy hypertension exactly. Preeclampsia is a distinct

cause of maternal morbidity and mortality and can lead to further systemic disorders. ${ }^{1}$ Future studies that address pre-eclampsia specifically are needed.

The strengths of our study include the following. Foremost, this is the first prospective study to investigate the long-term association between childhood abdominal obesity measures and pregnancy hypertension. Second, we conducted objective measurement of childhood anthropometrics including childhood BMI and abdominal obesity measures. Third, unlike 
previous two studies examining childhood BMI and pregnancy hypertension, a range of data on covariates has been collected in our study.

\section{Conclusion}

In conclusion, childhood abdominal obesity was associated with pregnancy hypertension independent of childhood and adult BMI. Women with persistently high adiposity from childhood to adulthood had the highest risk of pregnancy hypertension. Childhood abdominal obesity may be considered in addition to BMI, as an indicator of the risk of future cardiometabolic conditions including pregnancy hypertension. 


\section{Acknowledgments}

We gratefully acknowledge the contributions of the CDAH project manager, Marita Dalton, CDAH participants, funders and all other project staff. 


\section{References:}

1. DULEY L. The global impact of pre-eclampsia and eclampsia. Semin Perinatol 2009;33:130-7.

2. O'BRIEN TE, RAY JG, CHAN WS. Maternal body mass index and the risk of preeclampsia: a systematic overview. Epidemiology 2003;14:368-74.

3. CAMPBELL SK, LYNCH J, ESTERMAN A, MCDERMOTT R. Pre-pregnancy predictors of hypertension in pregnancy among Aboriginal and Torres Strait Islander women in north Queensland, Australia; a prospective cohort study. BMC Public Health 2013;13:138.

4. SINA M, HOY W, WANG Z. Anthropometric predictors of gestational hypertensive disorders in a remote aboriginal community: a nested case-control study. BMC research notes 2014;7:122.

5. LAKE JK, POWER C, COLE TJ. Women's reproductive health: the role of body mass index in early and adult life. Int J Obes Relat Metab Disord 1997;21:432-8.

6. LIS, XIONG X, HARVILLE E, et al. Childhood Risk Factors and Pregnancy-Induced Hypertension: The Bogalusa Heart Study. American journal of hypertension 2016;29:1206-11.

7. DWYER T, GIBBONS LE. The Australian Schools Health and Fitness Survey. Physical fitness related to blood pressure but not lipoproteins. Circulation 1994;89:1539-44.

8. COLE TJ, BeLLIZZI MC, FLEGAL KM, DIETZ WH. Establishing a standard definition for child overweight and obesity worldwide: international survey. BMJ 2000;320:1240-3.

9. BRAMBiLla P, BEDOGNI G, HEO M, PIETROBELLI A. Waist circumference-to-height ratio predicts adiposity better than body mass index in children and adolescents. International journal of obesity (2005) 2013;37:943-6.

10. VENN AJ, THOMSON RJ, SCHMIDT MD, et al. Overweight and obesity from childhood to adulthood: a follow-up of participants in the 1985 Australian Schools Health and Fitness Survey. Med J Aust 2007;186:458-60.

11. Obesity: preventing and managing the global epidemic. Report of a WHO consultation. World Health Organ Tech Rep Ser 2000;894:i-xii, 1-253.

12. BROWNING LM, HSIEH SD, ASHWELL M. A systematic review of waist-to-height ratio as a screening tool for the prediction of cardiovascular disease and diabetes: 0.5 could be a suitable global boundary value. Nutrition research reviews 2010;23:247-69.

13. PINK B. Socio-economic indexes for areas (SEIFA), technical paper, 2011: Canberra: Australian Bureau of Statistics, 2011.

14. Pare E, Parry S, McElrath TF, Pucci D, Newton A, Lim KH. Clinical risk factors for preeclampsia in the 21st century. Obstet Gynecol 2014;124:763-70.

15. Wallace M, Bazzano L, Chen W, Harville E. Maternal childhood cardiometabolic risk factors and pregnancy complications. Annals of epidemiology 2017;27:429-34.

16. SEAMAN SR, WHITE IR, COPAS AJ, LI L. Combining multiple imputation and inverse-probability weighting. Biometrics 2012;68:129-37.

17. BLUHER S, MOlz E, WIEGAND S, et al. Body mass index, waist circumference, and waist-toheight ratio as predictors of cardiometabolic risk in childhood obesity depending on pubertal development. The Journal of clinical endocrinology and metabolism 2013;98:3384-93.

18. LO K, WONG M, KHALECHELVAM P, TAM W. Waist-to-height ratio, body mass index and waist circumference for screening paediatric cardio-metabolic risk factors: a meta-analysis. Obesity reviews : an official journal of the International Association for the Study of Obesity 2016;17:1258-75.

19. SAVVA SC, TORNARITIS M, SAVVA ME, et al. Waist circumference and waist-to-height ratio are better predictors of cardiovascular disease risk factors in children than body mass index. Int J Obes Relat Metab Disord 2000;24:1453-8.

20. KeLSEY MM, ZAePFEL A, BJORNSTAD P, NADEAU KJ. Age-related consequences of childhood obesity. Gerontology 2014;60:222-8.

21. Callaway LK, O'Callaghan M, McInTYRe HD. Obesity and the hypertensive disorders of pregnancy. Hypertens Pregnancy 2009;28:473-93. 
22. RobertS JM, BOdnAR LM, PATRICK TE, POWERS RW. The Role of Obesity in Preeclampsia. Pregnancy Hypertens 2011;1:6-16.

23. SOLOMON CG, SEELY EW. Brief review: hypertension in pregnancy : a manifestation of the insulin resistance syndrome? Hypertension (Dallas, Tex : 1979) 2001;37:232-9.

24. ZAVALZA-GOMEZ AB. Obesity and oxidative stress: a direct link to preeclampsia? Archives of gynecology and obstetrics 2011;283:415-22.

25. DIETZ P, BOMBARD J, MULREADY-WARD C, et al. Validation of self-reported maternal and infant health indicators in the Pregnancy Risk Assessment Monitoring System. Matern Child Health J 2014;18:2489-98.

26. Coolman M, de Groot CJ, Jaddoe VW, Hofman A, RaAt H, Steegers EA. Medical record validation of maternally reported history of preeclampsia. Journal of clinical epidemiology 2010;63:932-7.

27. FaLKEGARD M, SCHIRMER H, LOCHEN ML, OIAN P, ACHARYA G. The validity of self-reported information about hypertensive disorders of pregnancy in a population-based survey: the Tromso Study. Acta obstetricia et gynecologica Scandinavica 2015;94:28-34. 
Table 1. Characteristic of participants in childhood (1985), CDAH-1 (2004-2006) and CDAH-2 (2009-2011), Childhood Determinants of Adult Health study.

Table 2. Associations between adiposity measures in childhood with pregnancy hypertension, Childhood Determinants of Adult Health study.

Table 3. Relative risk of pregnancy hypertension according to adiposity status from childhood to adulthood, Childhood Determinants of Adult Health study.

Figure 1. Selection of participants for the Childhood Determinants of Adult Health (CDAH) Study.

Figure 2. Percentage of ever had pregnancy hypertension across abdominal obesity category from childhood to adulthood, Childhood Determinants of Adult Health Study.

Supplemental Table 1. Associations between abdominal measures in childhood with pregnancy hypertension in CDAH-1 clinic, Childhood Determinants of Adult Health study.

Supplemental Table 2. Associations between adiposity measures in childhood with gestational hypertension, Childhood Determinants of Adult Health study (Considering childhood blood pressure).

Supplemental Table 3. Sensitivity analysis using inverse propensity weighting technique with multiple imputation. Associations between adiposity measures in childhood with pregnancy hypertension, Childhood Determinants of Adult Health Study. 
Table1. Characteristic of participants in childhood (1985), CDAH-1 (2004-2006) and CDAH-2 (2009-2011), Childhood Determinants of Adult Health study ${ }^{\mathrm{a}}$

\begin{tabular}{|c|c|c|c|}
\hline Characteristics & $\begin{array}{c}\text { Childhood } \\
(n=985)\end{array}$ & $\begin{array}{c}\text { CDAH-1 } \\
(n=806)\end{array}$ & $\begin{array}{c}\text { CDAH-2 } \\
(n=641)\end{array}$ \\
\hline Age, years, Mean(SD) & $12.1(2.0)$ & $32.8(2.0)$ & $37.5(2.0)$ \\
\hline BMI, kg/m², Mean(SD) & $18.8(2.8)$ & $25.0(5.1)$ & $25.2(5.5)$ \\
\hline Waist circumference, $\mathrm{cm}$, Mean(SD) & $64.1(7.5)$ & 79.3(10.9) & \\
\hline Waist-to-height ratio, Mean(SD) & $0.42(0.04)$ & $0.48(0.07)$ & \\
\hline Waist-to-hip ratio, Mean(SD) & $0.80(0.06)$ & $0.76(0.06)$ & \\
\hline \multicolumn{4}{|l|}{ BMI category $(\%)^{b}$} \\
\hline Normal & 91.2 & 60.7 & 59.9 \\
\hline Overweight & 7.8 & 24.8 & 22.9 \\
\hline Obese & 1.0 & 14.5 & 17.2 \\
\hline \multicolumn{4}{|l|}{ WHtR category $(\%)$} \\
\hline$<0.5$ & 95.2 & 69.0 & \\
\hline$\geq 0.5$ & 4.8 & 31.0 & \\
\hline \multicolumn{4}{|l|}{ Area-level disadvantage $(\%)$} \\
\hline High & 26.9 & 27.3 & 25.7 \\
\hline Medium-high & 28.9 & 25.0 & 24.7 \\
\hline Medium-low & 38.2 & 22.5 & 23.8 \\
\hline Low & 6.0 & 25.2 & 25.8 \\
\hline \multicolumn{4}{|l|}{ Highest parental education $(\%)$} \\
\hline University education & 23.3 & & \\
\hline Vocational training & 35.0 & & \\
\hline High school & 41.7 & & \\
\hline \multicolumn{4}{|l|}{ Childhood smoking experimentation (\%) } \\
\hline None & 56.1 & & \\
\hline A few puffs & 21.7 & & \\
\hline$<10$ cigarettes & 8.5 & & \\
\hline$>10$ cigarettes & 13.8 & & \\
\hline \multicolumn{4}{|l|}{ Parental smoking (\%) } \\
\hline None & 57.8 & & \\
\hline Either parent smoked & 26.8 & & \\
\hline Both parents smoked & 15.4 & & \\
\hline \multicolumn{4}{|l|}{ Childhood alcohol assumption (\%) } \\
\hline Never & 67.6 & & \\
\hline Less than once per week & 26.3 & & \\
\hline More than once per week & 6.1 & & \\
\hline Physical activity, mins/week, Mean(SD) ${ }^{\mathrm{c}}$ & $406.7(365.7)$ & $784.3(502.0)$ & $819.4(507.0)$ \\
\hline Systolic blood pressure, $\mathrm{mmHg}$, Mean(SD) & $108.9(12.0)$ & & \\
\hline \multicolumn{4}{|l|}{ Diastolic blood pressure, $\mathrm{mmHg}$} \\
\hline $\operatorname{Mean}(\mathrm{SD})$ & $66.6(11.0)$ & & \\
\hline \multicolumn{4}{|l|}{ Adulthood smoking status (\%) } \\
\hline Never smoker & & 49.7 & 56.1 \\
\hline Ex-smoker & & 28.0 & 30.4 \\
\hline Current smoker & & 22.4 & 13.5 \\
\hline Highest education attainment (\%) & & & \\
\hline
\end{tabular}


University education

Vocational training

High school

Adulthood alcohol consumption (\%)

Non-drinkers

Light drinkers

Moderate drinkers

Heavy/very heavy drinkers

Occupation (\%)

Professional or manager

Nonmanual

Manual

Not in the labor force

${ }^{a}$ Childhood overweight and obesity were defined according to the international cutoffs; the final sample $(n=985)$ included participants who participated in CDAH-1 $(n=806)$ or CDAH-2 ( $=641)$.

${ }^{\mathrm{b}}$ Sample size in Childhood ranges from 415-985, CDAH-1 ranges from 549-806, CDAH-2 ranges from 596-641 due to missing data on some variables;

${ }^{\mathrm{c}}$ Childhood physical activity was assessed by Australia Health and Fitness Survey, 1985; CDAH-1 and CDAH-2 physical activity was assessed by International Physical Activity Questionnaire;

Abbreviations: BMI, body mass index; CDAH, Childhood Determinants of Adult Health Study; SD, standard deviation; WHtR, waist-to-height ratio. 
Table 2 Associations between adiposity measures in childhood with pregnancy hypertension, Childhood Determinants of Adult Health study.

\begin{tabular}{|c|c|c|c|c|c|c|c|c|c|}
\hline \multirow[t]{2}{*}{ Body composition } & \multirow{2}{*}{$\mathrm{n}$} & \multicolumn{2}{|c|}{ Unadjusted model } & \multicolumn{2}{|c|}{ Model 1} & \multicolumn{2}{|c|}{ Model 2} & \multicolumn{2}{|c|}{ Model 3} \\
\hline & & RR & $95 \% \mathrm{CI}$ & RR & $95 \% \mathrm{CI}$ & $\mathrm{RR}$ & $95 \% \mathrm{CI}$ & RR & $95 \% \mathrm{CI}$ \\
\hline $\operatorname{BMI}\left(\mathrm{kg} / \mathrm{m}^{2}\right)$ & 985 & 1.06 & 1.01-1.12 & 1.08 & 1.02-1.14 & & & 1.04 & $0.97-1.11$ \\
\hline BMI z score & 985 & 1.19 & 1.03-1.38 & 1.20 & 1.04-1.40 & & & 1.09 & $0.92-1.30$ \\
\hline \multicolumn{10}{|l|}{ BMI category } \\
\hline Normal & 898 & Ref. & - & Ref. & - & & & Ref. & - \\
\hline Overweight & 77 & 1.68 & $1.06-2.67$ & 1.67 & $1.06-2.63$ & & & 1.31 & $0.79-2.17$ \\
\hline Obese & 10 & 1.27 & $0.30-5.30$ & 1.58 & $0.41-6.08$ & & & 1.00 & $0.29-3.39$ \\
\hline Waist circumference $(\mathrm{cm})$ & 985 & 1.03 & 1.01-1.05 & 1.04 & $1.02-1.06$ & 1.04 & 1.01-1.07 & 1.03 & $1.00-1.07$ \\
\hline Waist circumference $\mathrm{z}$ score & 985 & 1.28 & 1.11-1.41 & 1.29 & 1.12-1.49 & 1.28 & 1.01-1.61 & 1.25 & $0.99-1.57$ \\
\hline Waist circumference, per SD $(7.51 \mathrm{~cm})$ & 985 & 1.26 & 1.10-1.44 & 1.32 & 1.14-1.54 & 1.33 & 1.04-1.69 & 1.29 & 1.01-1.65 \\
\hline Waist-to-hip ratio, per SD $(0.06)$ & 985 & 1.22 & 1.05-1.42 & 1.25 & 1.07-1.47 & 1.22 & 1.04-1.43 & 1.20 & $1.03-1.41$ \\
\hline Waist-to-height ratio, per SD (0.04) & 985 & 1.33 & 1.17-1.51 & 1.33 & 1.15-1.53 & 1.35 & 1.09-1.67 & 1.24 & $1.06-1.43$ \\
\hline \multicolumn{10}{|l|}{ WHtR category } \\
\hline$<0.5$ & 938 & Ref. & - & Ref. & - & Ref. & - & Ref. & - \\
\hline$\geq 0.5$ & 47 & 2.33 & $1.45-3.74$ & 2.54 & $1.61-4.01$ & 2.14 & 1.18-3.87 & 2.21 & $1.09-4.48$ \\
\hline
\end{tabular}

Model 1: adjusted for age, area-level disadvantage at childhood, parity and occupation at adulthood;

Model 2: adjusted for age, area-level disadvantage and body mass index at childhood, parity and occupation at adulthood;

Model 3: adjusted for age, area-level disadvantage and body mass index at childhood (for abdominal obesity measures only), parity, occupation and body mass index at adulthood;

Abbreviations: BMI, body mass index; CI, confidence interval; RR, risk ratio; SD, standard deviation; WHtR, waist-to-height ratio. 
Table 3 Relative risk of pregnancy hypertension according to adiposity status from childhood to adulthood, Childhood Determinants of Adult Health Study.

Body composition from childhood to adulthood Pregnancy hypertension

\begin{tabular}{lllll}
$n(\%)^{\mathrm{a}}$ & \multicolumn{2}{l}{ Unadjusted model } & & \multicolumn{2}{c}{ Model 1} \\
\cline { 2 - 3 } \cline { 5 - 6 } & $\mathrm{RR} \quad 95 \% \mathrm{CI}$ & & $\mathrm{RR}$ & $95 \% \mathrm{CI}$ \\
\hline
\end{tabular}

BMI category $(\mathrm{N}=985)$

Persistently normal

Normal to overweight/obese

$\begin{array}{ccccc}860(59.4) & \text { Ref. } & - & \text { Ref. } & - \\ 469(32.4) & \mathbf{1 . 4 9} & \mathbf{1 . 1 1 - 2 . 0 1} & \mathbf{1 . 4 9} & \mathbf{1 . 1 0 - 2 . 0 2} \\ 13(0.9) & 1.00 & 0.15-6.53 & 1.09 & 0.16-7.29 \\ 105(7.3) & \mathbf{2 . 0 5} & \mathbf{1 . 2 7 - 3 . 2 9} & \mathbf{2 . 0 6} & \mathbf{1 . 2 9 - 3 . 2 9}\end{array}$

Persistently overweight/obese

WHtR category ${ }^{\mathrm{b}}(\mathrm{N}=549)$

Persistently not abdominally obese

375(68.3) Ref. - $\quad$ Ref.

Not abdominally obese to abdominally obese

$151(27.5)$

$4(0.7)$

1.56

1.00-2.43

Ref.

$19(3.5)$

$2.18 \quad 0.39-12.18$

1.43

$0.92-2.25$

Persistently abdominally obese

$2.75 \quad \mathbf{1 . 3 4 - 5 . 6 5}$

$2.13 \quad 0.40-11.43$

Model 1: adjusted for age, area-level disadvantage at childhood, parity and occupation at adulthood;

${ }^{a} \mathrm{n}$ indicated the total number of observations in each BMI category from childhood to CDAH-1 and/or

CDAH-2 and the number of participants in each WHtR category from childhood to CDAH-1;

${ }^{\mathrm{b}}$ Subgroup analysis which only available in those who participated in 1985 Australian Schools Health and Fitness Survey and CDAH-1 clinics;

Abbreviations: BMI, body mass index; RR, risk ratio; CI, confidence interval; WHtR, waist-height ratio. 
Supplemental Table 1 Associations between abdominal measures in childhood with pregnancy hypertension in CDAH-1 clinic, Childhood Determinants of Adult Health study.

\begin{tabular}{|c|c|c|c|c|c|c|c|}
\hline \multirow[t]{2}{*}{ Body composition } & \multirow{2}{*}{$\mathrm{n}$} & \multicolumn{2}{|c|}{ Unadjusted model } & \multicolumn{2}{|c|}{ Model 1} & \multicolumn{2}{|c|}{ Model 2} \\
\hline & & RR & $95 \% \mathrm{CI}$ & RR & $95 \% \mathrm{CI}$ & RR & $95 \% \mathrm{CI}$ \\
\hline Waist circumference $(\mathrm{cm})$ & 549 & 1.03 & $1.00-1.06$ & 1.04 & 1.01-1.07 & 1.03 & $1.00-1.07$ \\
\hline Waist circumference $\mathrm{z}$ score & 549 & 1.26 & 1.03-1.55 & 1.33 & 1.08-1.65 & 1.22 & $0.96-1.56$ \\
\hline Waist circumference, per SD $(7.30 \mathrm{~cm})$ & 549 & 1.22 & 1.01-1.48 & 1.36 & 1.09-1.69 & 1.25 & $0.97-1.60$ \\
\hline Waist-to-hip ratio, per SD (0.06) & 549 & 1.15 & $0.93-1.42$ & 1.21 & $0.96-1.52$ & 1.20 & $0.94-1.52$ \\
\hline Waist-to-height ratio, per SD (0.04) & 549 & 1.32 & 1.10-1.58 & 1.34 & 1.11-1.61 & 1.24 & 1.01-1.54 \\
\hline \multicolumn{8}{|l|}{ WHtR category } \\
\hline$<0.5$ & 526 & Ref. & - & Ref. & - & Ref. & - \\
\hline$\geq 0.5$ & 23 & 2.29 & $1.19-4.40$ & 2.55 & 1.34-4.85 & 2.15 & $1.10-4.20$ \\
\hline
\end{tabular}

Model 1: adjusted for age, area-level disadvantage at childhood, parity and occupation at adulthood; Model 2: adjusted for age, area-level disadvantage at childhood, parity, occupation and corresponding abdominal measures at adulthood;

Abbreviations: CI, confidence interval; RR, risk ratio; SD, standard deviation; WHtR, waist-to-height ratio. 
Supplemental Table 2 Associations between adiposity measures in childhood with pregnancy hypertension, Childhood Determinants of Adult Health Study (Considering childhood blood pressure).

\begin{tabular}{|c|c|c|c|c|c|c|c|c|c|c|c|}
\hline \multirow[t]{2}{*}{ Body composition } & \multirow{2}{*}{$\mathrm{n}$} & \multicolumn{2}{|c|}{ Unadjusted model } & \multicolumn{2}{|c|}{ Model 1} & \multicolumn{2}{|c|}{ Model 2} & \multicolumn{2}{|c|}{ Model 3} & \multicolumn{2}{|c|}{ Model 4} \\
\hline & & $\mathrm{RR}$ & $95 \% \mathrm{CI}$ & $\mathrm{RR}$ & $95 \% \mathrm{CI}$ & RR & $95 \% \mathrm{CI}$ & RR & $95 \% \mathrm{CI}$ & RR & $95 \% \mathrm{CI}$ \\
\hline $\mathrm{BMI}\left(\mathrm{kg} / \mathrm{m}^{2}\right)$ & 415 & 1.10 & $1.02-1.18$ & 1.12 & $1.03-1.21$ & & & 1.03 & $0.93-1.15$ & 1.04 & $0.93-1.16$ \\
\hline BMI z score & 415 & 1.29 & $1.04-1.58$ & 1.31 & $1.06-1.62$ & & & 1.06 & $0.81-1.40$ & 1.09 & $0.81-1.44$ \\
\hline \multicolumn{12}{|l|}{ BMI category } \\
\hline Normal & 380 & Ref. & - & Ref. & - & & & Ref. & - & Ref. & - \\
\hline Overweight/obese & 35 & 2.58 & $1.50-4.45$ & 2.75 & $1.60-4.73$ & & & 1.85 & $0.95-3.59$ & 1.98 & $0.91-4.30$ \\
\hline Waist circumference $(\mathrm{cm})$ & 415 & 1.04 & 1.02-1.07 & 1.05 & 1.02-1.08 & 1.05 & $1.00-1.09$ & 1.04 & $1.00-1.09$ & 1.04 & 1.01-1.09 \\
\hline Waist circumference z score & 415 & 1.38 & 1.14-1.67 & 1.41 & 1.17-1.70 & 1.36 & $0.99-1.87$ & 1.35 & 1.00-1.82 & 1.36 & $1.03-1.80$ \\
\hline Waist circumference, per SD $(7.87 \mathrm{~cm})$ & 415 & 1.38 & 1.15-1.66 & 1.46 & $1.20-1.78$ & 1.42 & 1.01-2.01 & 1.41 & 1.04-1.92 & 1.43 & 1.07-1.90 \\
\hline Waist-to-hip ratio, per SD $(0.06)$ & 415 & 1.26 & $1.00-1.58$ & 1.33 & $1.04-1.70$ & 1.25 & $0.98-1.60$ & 1.23 & $0.97-1.57$ & 1.23 & $0.97-1.56$ \\
\hline Waist-to-height ratio, per SD (0.04) & 415 & 1.43 & $1.20-1.71$ & 1.44 & $1.22-1.70$ & 1.44 & $1.09-1.90$ & 1.41 & $1.10-1.81$ & 1.40 & $1.09-1.80$ \\
\hline \multicolumn{12}{|l|}{ WHtR category } \\
\hline$<0.5$ & 395 & Ref. & - & Ref. & - & Ref. & - & Ref. & - & Ref. & - \\
\hline$\geq 0.5$ & 20 & 3.24 & 1.81-5.80 & 3.50 & 2.01-6.10 & 2.88 & $1.25-6.59$ & 2.75 & 1.11-6.82 & 3.28 & 1.56-6.87 \\
\hline
\end{tabular}

Model 1: adjusted for age, area-level disadvantage at childhood, parity and occupation at adulthood;

Model 2: adjusted for age, area-level disadvantage and body mass index at childhood, parity and occupation at adulthood;

Model 3: adjusted for age, area-level disadvantage and body mass index at childhood (for abdominal obesity measures only), parity, occupation and body mass index at adulthood;

Model 4: adjusted for age, area-level disadvantage, body mass index (for abdominal obesity measures only) and systolic and diastolic blood pressure at childhood, parity, occupation and body mass index at adulthood;

Abbreviations: BMI, body mass index; CI, confidence interval; RR, risk ratio; SD, standard deviation; WHtR, waist-to-height ratio. 
Supplemental Table 3 Sensitivity analysis using inverse propensity weighting technique with multiple imputation. Associations between adiposity measures in childhood with pregnancy hypertension, Childhood Determinants of Adult Health Study.

\begin{tabular}{|c|c|c|c|c|c|c|c|c|c|}
\hline \multirow[t]{2}{*}{ Body composition } & \multirow{2}{*}{$\mathrm{n}$} & \multicolumn{2}{|c|}{ Unadjusted model } & \multicolumn{2}{|c|}{ Model 1} & \multicolumn{2}{|c|}{ Model 2} & \multicolumn{2}{|c|}{ Model 3} \\
\hline & & $\mathrm{RR}$ & $95 \% \mathrm{CI}$ & RR & $95 \% \mathrm{CI}$ & RR & $95 \% \mathrm{CI}$ & RR & $95 \% \mathrm{CI}$ \\
\hline BMI $\left(\mathrm{kg} / \mathrm{m}^{2}\right)$ & 985 & 1.06 & 1.01-1.12 & 1.07 & 1.01-1.13 & & & 1.02 & $0.96-1.09$ \\
\hline BMI z score & 985 & 1.18 & 1.01-1.38 & 1.19 & 1.02-1.39 & & & 1.04 & $0.87-1.24$ \\
\hline \multicolumn{10}{|l|}{ BMI category } \\
\hline Normal & 898 & Ref. & - & Ref. & - & & & Ref. & - \\
\hline Overweight & 77 & 1.72 & 1.07-2.77 & 1.76 & $1.10-2.82$ & & & 1.22 & $0.73-2.05$ \\
\hline Obese & 10 & 1.22 & $0.33-4.45$ & 1.26 & $0.35-4.51$ & & & 0.50 & $0.13-1.88$ \\
\hline Waist circumference $(\mathrm{cm})$ & 985 & 1.03 & 1.01-1.05 & 1.04 & 1.02-1.06 & 1.04 & 1.01-1.07 & 1.03 & $1.00-1.07$ \\
\hline Waist circumference z score & 985 & 1.28 & 1.10-1.48 & 1.28 & $1.10-1.48$ & 1.28 & $1.02-1.60$ & 1.21 & $0.94-1.57$ \\
\hline Waist circumference, per SD $(7.51 \mathrm{~cm})$ & 985 & 1.26 & 1.10-1.45 & 1.31 & $1.12-1.52$ & 1.32 & $1.05-1.68$ & 1.26 & $0.97-1.65$ \\
\hline Waist-to-hip ratio, per SD (0.06) & 985 & 1.22 & 1.04-1.44 & 1.29 & 1.09-1.52 & 1.25 & 1.05-1.49 & 1.26 & 1.05-1.50 \\
\hline Waist-to-height ratio, per SD (0.04) & 985 & 1.33 & 1.17-1.51 & 1.33 & 1.18-1.51 & 1.43 & 1.18-1.74 & 1.40 & 1.3-1.72 \\
\hline \multicolumn{10}{|l|}{ WHtR category } \\
\hline$<0.5$ & 938 & Ref. & - & Ref. & - & Ref. & - & Ref. & - \\
\hline$\geq 0.5$ & 47 & 2.36 & $1.46-3.82$ & 2.48 & $1.54-4.00$ & 2.15 & $1.13-4.08$ & 2.50 & $1.43-4.37$ \\
\hline
\end{tabular}

Model 1: adjusted for age, area-level disadvantage at childhood, parity and occupation at adulthood;

Model 2: adjusted for age, area-level disadvantage and body mass index at childhood, parity and occupation at adulthood;

Model 3: adjusted for age, area-level disadvantage and body mass index (for abdominal obesity measures only) at childhood, parity, occupation and body mass index at adulthood;

Abbreviations: BMI, body mass index; CI, confidence interval; RR, risk ratio; SD, standard deviation; WHtR, waist-to-height ratio. 


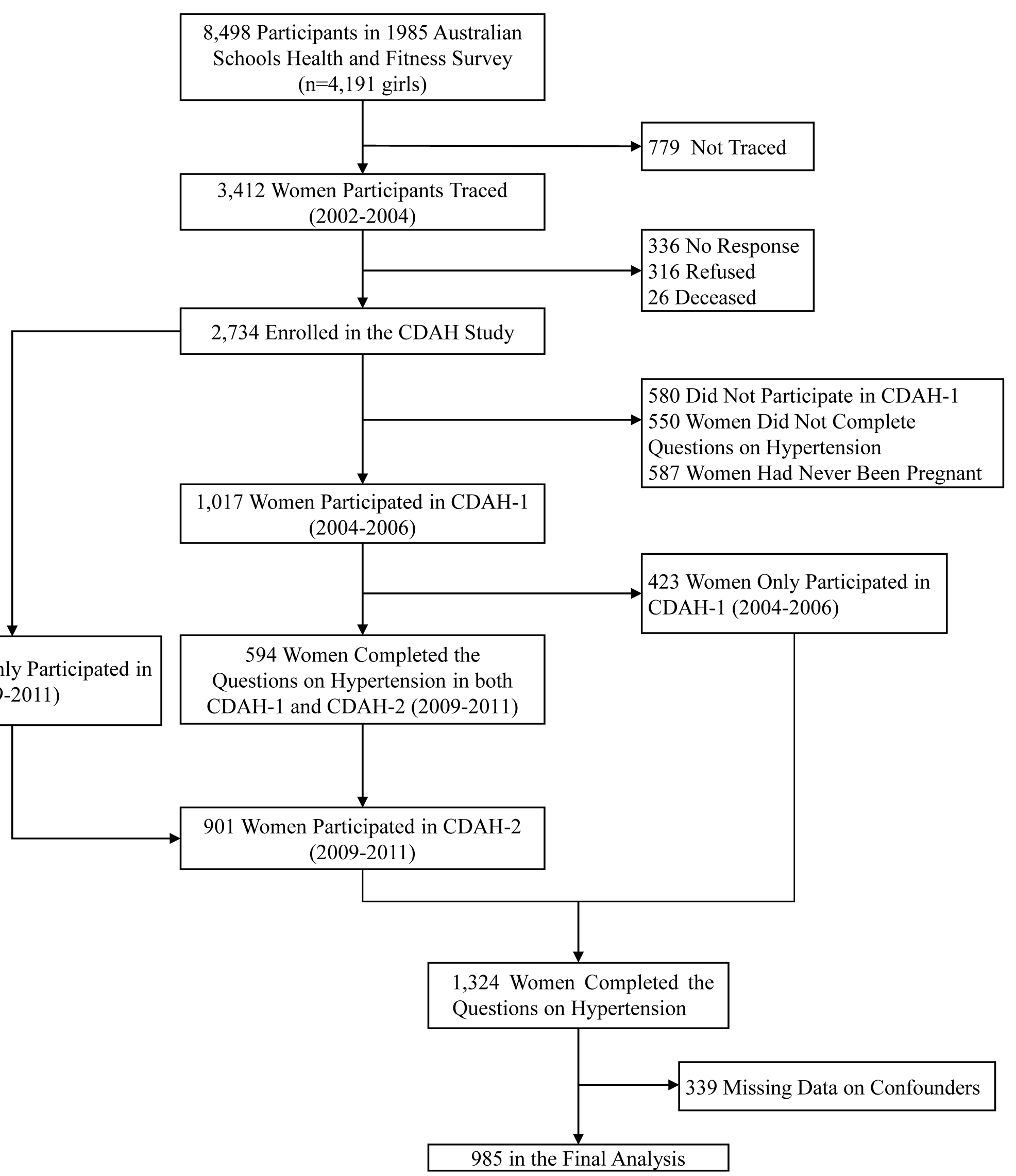




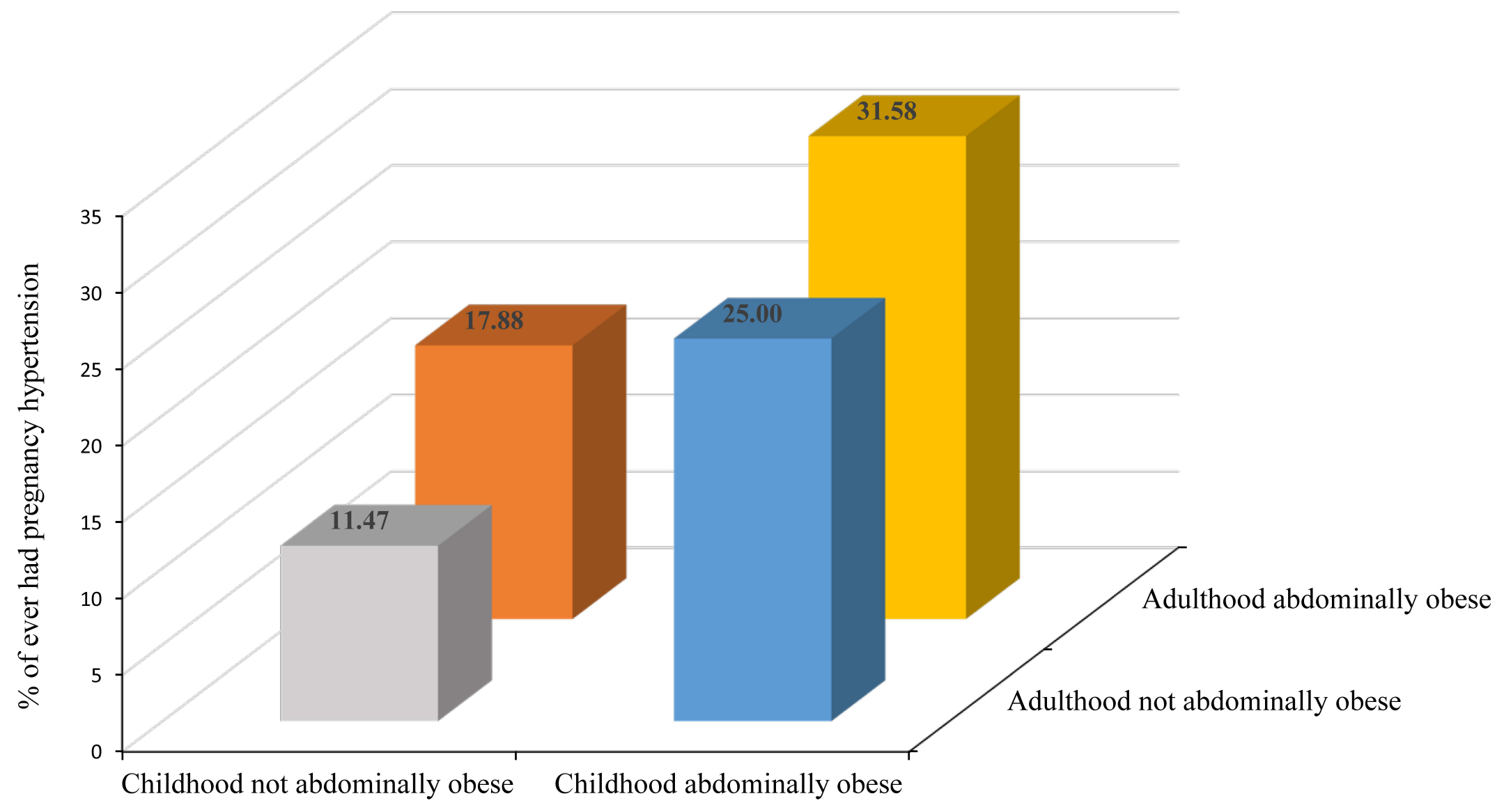

